PROCEEDINGS OF THE

AMERICAN MATHEMATICAL SOCIETY

Volume 133, Number 3, Pages 727-734

S 0002-9939(04)07715-9

Article electronically published on October 21, 2004

\title{
PERTURBED SMOOTH LIPSCHITZ EXTENSIONS OF UNIFORMLY CONTINUOUS FUNCTIONS ON BANACH SPACES
}

\author{
DANIEL AZAGRA, ROBB FRY, AND ALEJANDRO MONTESINOS
}

(Communicated by Jonathan M. Borwein)

\begin{abstract}
We show that if $Y$ is a separable subspace of a Banach space $X$ such that both $X$ and the quotient $X / Y$ have $C^{p}$-smooth Lipschitz bump functions, and $U$ is a bounded open subset of $X$, then, for every uniformly continuous function $f: Y \cap U \rightarrow \mathbb{R}$ and every $\varepsilon>0$, there exists a $C^{p}$-smooth Lipschitz function $F: X \rightarrow \mathbb{R}$ such that $|F(y)-f(y)| \leq \varepsilon$ for every $y \in Y \cap U$.
\end{abstract}

If we are given a separable subspace $Y$ of a Banach space $X$ and a continuous (resp. Lipschitz) function $f: Y \rightarrow \mathbb{R}$, under what conditions can we ensure the existence of a $C^{p}$-smooth (Lipschitz) perturbed extension of $f$ ? That is, for a given $\varepsilon>0$, does there exist a $C^{p}$-smooth (Lipschitz) function $F: X \rightarrow \mathbb{R}$ so that $|F(y)-f(y)| \leq \varepsilon$ for all $y \in Y$ ? Such an $F$ will be called a smooth perturbed extension of $f$.

Of course there are several conditions under which the answer is "yes" in a trivial way (for the non-Lipschitz case). For instance, when $X$ has $C^{p}$-smooth partitions of unity, and also when $Y$ has a $C^{p}$-smooth bump function and the subspace $Y$ is complemented in $X$, such perturbed extensions $F$ are easily proved to exist. However, in the Lipschitz case, or when the space $X$ does not have smooth partitions of unity and $Y$ is not complemented in $X$, it is not quite clear what the answer is.

These questions are interesting in the theory of Banach spaces because, for instance, while trying to prove a theorem, one might be able to construct a continuous (resp. Lipschitz) function $f$ with some nice properties, but defined only on a certain separable subspace $Y$ of $X$, and then one might want to obtain a smooth (resp. and Lipschitz) function $F$ defined on the whole of $X$ that behaves on $Y$ almost the same way as $f$ does.

In this paper we will try to give a solution to the above question in the Lipschitz case. This problem is clearly related to the question concerning uniform approximation of Lipschitz functions by smooth Lipschitz functions on infinite-dimensional spaces, which has remained unasked and open until recent times: in 7] R. Fry has shown that, on any separable Banach space with a $C^{1}$-smooth bump function, uniformly continuous functions can be approximated by $C^{1}$-smooth Lipschitz functions, uniformly on bounded sets.

Received by the editors January 26, 2003.

2000 Mathematics Subject Classification. Primary 46B20.

(C)2004 American Mathematical Society Reverts to public domain 28 years from publication 
We will use some of the ideas of the proof of [7, combined with some techniques for starlike bodies, to prove the following theorem, which provides a solution to the above "smooth perturbed extension" problem.

Theorem 1. Let $\left(X,\|\cdot\|_{X}\right)$ be a Banach space and $Y$ a separable subspace of $X$ such that both $X$ and $X / Y$ admit a Lipschitz $C^{p}$-smooth bump function, where $p \in \mathbb{N} \cup\{\infty\}$. Let us consider $U$, an open subset of $X$, and a bounded uniformly continuous function $f: Y \cap U \rightarrow \mathbb{R}$. Then, for every $\varepsilon>0$ there exists a $C^{p}$-smooth Lipschitz function $K: X \rightarrow \mathbb{R}$ such that $|K(x)-f(x)| \leq \varepsilon$ for all $x \in Y \cap U$.

Here $p \in \mathbb{N} \cup\{\infty\}$. Note that, if we take $X=Y$, we obtain a generalization of the main result of [7] (which was proved only for $p=1$ ). Note also that if the open set $U$ is assumed to be bounded, then it is not necessary to demand that $f$ be bounded, because every uniformly continuous function is bounded on bounded sets.

As we have already said, starlike bodies and their Minkowski functionals will play an important role in our proof. Let us recall the definition and some of the elementary properties of starlike bodies. A closed subset $A$ of a Banach space $X$ is said to be a starlike body if there exists a point $a_{0}$ in the interior of $A$ such that every ray emanating from $a_{0}$ meets $\partial A$, the boundary of $A$, at most once. We will say that $a_{0}$ is a center of $A$. There can obviously exist many centers for a given starlike body. Up to a suitable translation, we can always assume that $a_{0}=0$ is the origin of $X$, and we will often do so, unless otherwise stated. For a starlike body $A$ with center $a_{0}$, we define the characteristic cone of $A$ as

$$
c c A=\left\{x \in X \mid a_{0}+r\left(x-a_{0}\right) \in A \text { for all } r>0\right\},
$$

and the Minkowski functional of $A$ with respect to the center $a_{0}$ as

$$
\mu_{A, a_{0}}(x)=\mu_{A}(x)=\inf \left\{t>0 \mid x-a_{0} \in t\left(-a_{0}+A\right)\right\} \text { for all } x \in X .
$$

Note that $\mu_{A}(x)=\mu_{-a_{0}+A}\left(x-a_{0}\right)$ for all $x \in X$. It is easily seen that $\mu_{A}$ is a continuous function that satisfies $\mu_{A}\left(a_{0}+r x\right)=r \mu_{A}\left(a_{0}+x\right)$ for every $r \geq 0$ and $x \in X$, and $\mu_{A}^{-1}(0)=c c A$. Moreover, $A=\left\{x \in X \mid \mu_{A}(x) \leq 1\right\}$, and $\partial A=$ $\left\{x \in X \mid \mu_{A}(x)=1\right\}$. Conversely, if $\psi: X \rightarrow[0, \infty)$ is continuous and satisfies $\psi\left(a_{0}+\lambda x\right)=\lambda \psi\left(a_{0}+x\right)$ for all $\lambda \geq 0$, then $A_{\psi}=\{x \in X \mid \psi(x) \leq 1\}$ is a starlike body.

We will say that $A$ is a $C^{p}$-smooth starlike body provided its Minkowski functional $\mu_{A}$ is $C^{p}$-smooth on the set $X \backslash c c A=X \backslash \mu_{A}^{-1}(0)$. This is equivalent to saying that $\partial A$ is a $C^{p}$-smooth one-codimensional submanifold of $X$ such that no affine hyperplane tangent to $\partial A$ contains a ray emanating from the center $a_{0}$. Throughout this paper, $p=1,2, \ldots, \infty$. Similarly, we will say that a starlike body $A$ is Lipschitz if $\mu_{A}$ is Lipschitz on $X$.

The following result is implicitly proved in [6, Proposition II.5.1]; see also [8].

Proposition 2. Let $X$ be a Banach space and $p \in \mathbb{N} \cup\{\infty\}$. The following assertions are equivalent.

(1) $X$ admits a $C^{p}$-smooth Lipschitz bump function.

(2) There exist numbers $a, b>0$ and a Lipschitz function $\psi: X \rightarrow[0, \infty)$ which is $C^{p}$-smooth on $X \backslash\{0\}$, homogeneous (that is $\psi(t x)=|t| \psi(x)$ for all $t \in \mathbb{R}, x \in X)$, and such that $a\|\cdot\| \leq \psi \leq b\|\cdot\|$.

(3) There exists a symmetric bounded $C^{p}$-smooth Lipschitz starlike body E with center $0 \in X$. 
As a consequence we can deduce the following result, which plays a technical but fundamental role in our proof.

Corollary 3. Let $X$ be a Banach space, $Y$ a closed subspace of $X$, and $p \in \mathbb{N} \cup\{\infty\}$. Consider the following assertions.

(1) $X / Y$ admits a $C^{p}$-smooth Lipschitz bump function.

(2) There exist numbers $a, b>0$ and a Lipschitz function $\psi: X \rightarrow[0, \infty)$ that is homogeneous, $C^{p}$-smooth on $X \backslash Y$, and such that a $\operatorname{dist}(x, Y) \leq \psi(x) \leq$ $b \operatorname{dist}(x, Y)$ for all $x \in X$.

(3) There exists a symmetric $C^{p}$-smooth Lipschitz starlike body $E$ with center $0 \in X$ and whose characteristic cone is $Y$.

Then $(1) \Longrightarrow(2) \Longrightarrow(3)$.

Proof. We denote by $|\cdot|$ the canonical norm on $X / Y$, and let $\pi$ be the canonical quotient map of $X$ onto $X / Y$. If (1) is true, then, according to Proposition 2, there exist positive real numbers $a, b$ and a Lipschitz function $\Psi: X / Y \rightarrow[0, \infty)$, that is homogeneous, $C^{p}$-smooth on $X / Y \backslash\{0\}$, and such that $a|\cdot| \leq \Psi \leq b|\cdot|$. Then the function $\psi=\Psi \circ \pi$ makes (2) hold true. To see that (2) $\Longrightarrow$ (3) it is enough to define $E$ as the set $\{x \in X: \psi(x) \leq 1\}$.

Proof of Theorem 1. Without loss of generality, and taking into account the wellknown fact that every uniformly continuous function can be uniformly approximated by Lipschitz functions (see [2] for instance), we may assume that $f$ is Lipschitz.

Moreover, since $f$ is bounded and Lipschitz on $Y \cap U$, there exists a bounded Lipschitz function $F: Y \rightarrow \mathbb{R}$ that is an extension of $f$. Indeed, let $L$ be the Lipschitz constant of $f$ on $Y \cap U$, and let $C>0$ be such that $|f(x)|+1 \leq C$. The function $g: Y \rightarrow \mathbb{R}$ defined as

$$
g(y)=\inf \{f(u)+L\|y-u\|: u \in Y \cap U\}
$$

is easily seen to be a Lipschitz extension of $f$ to $Y$. Then, the function $F: Y \rightarrow \mathbb{R}$ defined by

$$
F(y)=\left\{\begin{array}{ccc}
-C & \text { if } & g(y) \leq-C \\
g(y) & \text { if } & -C \leq g(y) \leq C \\
C & \text { if } & C \leq g(y)
\end{array}\right.
$$

is a bounded Lipschitz extension of $f$ to $Y$.

Since $f$ and $F$ are bounded, we may obviously assume that $F, f \geq 1$.

From the assumption that $X$ and $X / Y$ have $C^{p}$-smooth Lipschitz bumps we infer two facts. First, the existence of a symmetric $C^{p}$-smooth Lipschitz starlike body $B_{X} \subset E$ with center the origin (see Proposition 2). Therefore the Minkowski functional $\mu_{E}: X \rightarrow[0, \infty)$ is Lipschitz, homogeneous, and $C^{p}$-smooth on $X \backslash\{0\}$. Second (see Corollary 3), there are a Lipschitz function $\rho: X \rightarrow[0, \infty)$ and a real number $M>1$ such that

(1) $\rho(t x)=|t| \rho(x)$ for all $t \in \mathbb{R}, x \in X$;

(2) $\rho^{-1}(0)=Y$;

(3) $\rho$ is $C^{p}$-smooth on $X \backslash Y$;

(4) $\operatorname{dist}(x, Y) \leq \rho(x) \leq M \operatorname{dist}(x, Y)$, for all $x \in X$.

Let $\left(e_{i}\right)_{i=1}^{\infty}$ be the usual basis of $c_{0}$. Let us consider the function $\Phi: c_{0} \rightarrow \mathbb{R}$ defined by $\Phi(x)=\sum_{n=1}^{\infty} x_{n}^{2 n}$ for each $x=\left(x_{n}\right)_{n=1}^{\infty} \in c_{0}$. The set $A=\left\{x \in c_{0}: \Phi(x) \leq 1\right\}$ 
is closed, convex, bounded and symmetric with respect to the origin (which is contained as an interior point). Hence $\|\cdot\|:=\mu_{A}(\cdot)$ is a $C^{\infty}$-smooth (in fact realanalytic) norm equivalent to the canonical norm $\|\cdot\|_{\infty}$ (see [6. Theorem V.1.5 and Remark V.1.6]). It is easy to verify that $\frac{1}{2}\|\cdot\| \leq\|\cdot\|_{\infty} \leq\|\cdot\|$. Moreover, $\|\cdot\|$ has the following nice property.

Fact 4. If $x=\left(x_{n}\right)_{n=1}^{\infty}, y=\left(y_{n}\right)_{n=1}^{\infty} \in c_{0}$ satisfy $\left|x_{n}\right| \leq\left|y_{n}\right|$ for every $n \in \mathbb{N}$, then $\|x\| \leq\|y\|$.

Proof. For every $\lambda>0$ we have that

$$
\sum_{n=1}^{\infty}\left(\frac{x_{n}}{\lambda}\right)^{2 n} \leq \sum_{n=1}^{\infty}\left(\frac{y_{n}}{\lambda}\right)^{2 n} .
$$

It follows that $\inf \{\lambda>0: \Phi(y / \lambda) \leq 1\} \leq \inf \{\lambda>0: \Phi(x / \lambda) \leq 1\}$, that is, $\|x\| \leq\|y\|$.

Fix $\varepsilon \in(0,1)$. Since $F: Y \rightarrow \mathbb{R}$ is Lipschitz and, in particular, uniformly continuous, there exists $\delta>0$ such that, for all $x, y \in Y$, we have

$$
\mu_{E}(x-y) \leq \delta \Rightarrow|F(x)-F(y)| \leq \varepsilon .
$$

For every $x \in X$ and $r>0$, the set

$$
B\left(x, r ; \mu_{E}\right)=\left\{y \in X: \mu_{E}(y-x)<r\right\}
$$

is open. By the separability of $Y$, we can take a dense sequence $\left(x_{j}\right)_{j=1}^{\infty}$ in $Y$. We have that

$$
Y \subset \bigcup_{j=1}^{\infty} B\left(x_{j}, \frac{\delta}{8} ; \mu_{E}\right) .
$$

Let us define the open subset $V:=\bigcup_{j=1}^{\infty} B\left(x_{j}, \frac{\delta}{8} ; \mu_{E}\right)$. In the sequel, we will say that a family of functions $\left\{h_{i}\right\}_{i \in I}$ is uniformly Lipschitz if there is a number $C>0$ so that $h_{i}$ is $C$-Lipschitz for every $i \in I$.

Claim 5. There exists a family $\left\{\Psi_{j}: j \in \mathbb{N}\right\}$ of non-negative $C^{p}$-smooth functions defined on $X$ such that:

(i) $\left\{\Psi_{j}: j \in \mathbb{N}\right\}$ is uniformly Lipschitz and uniformly bounded.

(ii) For every $x \in V$, there exist $W_{x}$, an open neighborhood of $x \in V$, and $j_{x} \in \mathbb{N}$, such that $\left.\Psi_{j}\right|_{W_{x}}=0$ if $j>j_{x}$.

(iii) For every $x \in V$, there exists $j_{0} \in \mathbb{N}$ such that $\Psi_{j_{0}}(x)=1$.

(iv) For every $x \in V$ and $j \in \mathbb{N}$, if $\mu_{E}\left(x-x_{j}\right) \geq \delta$, then $\Psi_{j}(x)=0$.

Let us assume for a while that Claim 5 is proved and let us see how the proof of Theorem 1 is finished.

By (ii) of Claim [5] for every $x \in V$ there exist $W_{x}$, an open neighborhood of $x \in V$, and $j_{x} \in \mathbb{N}$ such that

$$
y \in W_{x}, j>j_{x} \Rightarrow \Psi_{j}(y)=0 .
$$

Then the mapping

$$
V \ni x \longrightarrow\left\{\Psi_{j}(x)\right\}_{j=1}^{\infty} \in c_{00} \subset c_{0}
$$

locally takes values on finite-dimensional subspaces of $c_{00}$. Clearly it is also Lipschitz and $C^{p}$-smooth (note $(i)$ of Claim 5). Let us define $\Psi: V \rightarrow \mathbb{R}$ by

$$
\Psi(x)=\left\|\left\{\Psi_{j}(x)\right\}_{j=1}^{\infty}\right\|
$$


which is a Lipschitz function. To prove that $\Psi$ is $C^{p}$-smooth, it suffices to check that $\Psi$ is bounded below by a strictly positive real number. Indeed, by (iii) of Claim [5, for each $x \in V$, there exists $j_{0} \in \mathbb{N}$ such that $\Psi_{j_{0}}(x)=1$, and thus

$$
\Psi(x)=\left\|\left\{\Psi_{j}(x)\right\}_{j=1}^{\infty}\right\| \geq\left\|\left\{\Psi_{j}(x)\right\}_{j=1}^{\infty}\right\|_{\infty} \geq \Psi_{j_{0}}(x)=1 .
$$

It follows that $\Psi$ is a $C^{p}$-smooth function and $\Psi \geq 1$.

Define then $K_{0}: V \rightarrow[0, \infty)$ by

$$
K_{0}(x)=\frac{\left\|\left\{F\left(x_{j}\right) \Psi_{j}(x)\right\}_{j=1}^{\infty}\right\|}{\Psi(x)} .
$$

Taking into account that $F \geq 1$, it is obvious that

$$
\left\|\left\{F\left(x_{j}\right) \Psi_{j}(x)\right\}_{j=1}^{\infty}\right\| \geq\left\|\left\{F\left(x_{j}\right) \Psi_{j}(x)\right\}_{j=1}^{\infty}\right\|_{\infty} \geq\left\|\left(\Psi_{j}(x)\right)_{j=1}^{\infty}\right\|_{\infty} \geq 1 .
$$

Therefore $K_{0}$ is $C^{p}$-smooth. Let us see that $K_{0}$ is a Lipschitz function. Since $F$ is bounded, and the family $\left\{\Psi_{j}: j \in \mathbb{N}\right\}$ is uniformly bounded, it is obvious that the collection $\left\{F\left(x_{j}\right) \Psi_{j}: j \in \mathbb{N}\right\} \subset C^{p}(V)$ is uniformly Lipschitz and uniformly bounded, which implies that the numerator of $K_{0}$ is bounded and Lipschitz. Besides, since $\Psi$ is Lipschitz and $\Psi \geq 1$, it follows that $K_{0}$ is Lipschitz (note that the inverse of a Lipschitz function bounded below by a strictly positive real number is Lipschitz).

Finally let us see that $\left|K_{0}(x)-F(x)\right| \leq \varepsilon$ for every $x \in Y$. Fix $x \in Y$. Then,

$$
\begin{aligned}
& |K(x)-F(x)|=\left|\frac{\left\|\left\{F\left(x_{j}\right) \Psi_{j}(x)\right\}_{j=1}^{\infty}\right\|}{\Psi(x)}-F(x)\right| \\
& =\left|\frac{\left\|\left\{F\left(x_{j}\right) \Psi_{j}(x)\right\}_{j=1}^{\infty}\right\|}{\Psi(x)}-\frac{F(x) \Psi(x)}{\Psi(x)}\right|=\frac{1}{\Psi(x)}\left\|\left\{\Psi_{j}(x)\left(F\left(x_{j}\right)-F(x)\right)\right\}_{j=1}^{\infty}\right\| .
\end{aligned}
$$

Let us consider the set $J:=\left\{j \in \mathbb{N}: \mu_{E}\left(x-x_{j}\right)<\delta\right\}$. We can distinguish two situations.

Case 1. If $j \in J$, then $\mu_{E}\left(x-x_{j}\right)<\delta$, and thus $\left|F(x)-F\left(x_{j}\right)\right| \leq \varepsilon$.

Case 2. Assume that $j \notin J$. Then $\mu_{E}\left(x-x_{j}\right) \geq \delta$ and, by (iv) of Claim 5 we have $\Psi_{j}(x)=0$.

By collecting the conclusions of the distinguished cases, we deduce that

$$
\Psi_{j}(x)\left|F\left(x_{j}\right)-F(x)\right| \leq \varepsilon \Psi_{j}(x)
$$

for every $j \geq 1$. Since $\left\{\Psi_{j}(x)\left(F\left(x_{j}\right)-F(x)\right)\right\}_{j=1}^{\infty} \in c_{00}$, taking Fact 4 into account we may estimate

$$
\begin{aligned}
& \left|K_{0}(x)-F(x)\right|=\frac{1}{\Psi(x)}\left\|\left\{\Psi_{j}(x)\left(F\left(x_{j}\right)-F(x)\right)\right\}_{j=1}^{\infty}\right\| \\
& \leq \frac{1}{\Psi(x)}\left\|\varepsilon\left\{\Psi_{j}(x)\right\}_{j=1}^{\infty}\right\|=\varepsilon \frac{\Psi(x)}{\Psi(x)}=\varepsilon .
\end{aligned}
$$

Hence $K_{0}: V \rightarrow \mathbb{R}$ is a Lipschitz $C^{p}$-smooth function such that $\left|K_{0}(x)-F(x)\right| \leq \varepsilon$ for every $x \in Y$. 
Finally, let us see how we can extend the function $K_{0}$ from the open set $V$ to the whole of $X$. Since $B_{X} \subset E$, there exists a real number $m \geq 1$ that satisfies $m\|\cdot\|_{X} \leq \mu_{E} \leq\|\cdot\|_{X}$. By property (4) of $\rho$, it is easy to see that

$$
Y \subset\left\{x \in X: \rho(x)<\frac{\delta}{8}\right\} \subset V \subset\left\{x \in X: \rho(x)<\frac{M \delta}{8 m}\right\} .
$$

Let us pick two positive real numbers $r_{1}$ and $r_{2}$ such that $0<r_{1}<r_{2}<\frac{\delta}{8}$. Take a $C^{\infty}$-smooth function $\theta: \mathbb{R} \rightarrow[0,1]$ such that $\theta^{-1}(1)=\left(-\infty, r_{1}\right]$ and $\theta^{-1}(0)=$ $\left[r_{2}, \infty\right)$. Then, the function $K: X \rightarrow \mathbb{R}$ defined by

$$
K(x)=\left\{\begin{array}{l}
\theta(\rho(x)) K_{0}(x) \text { if } x \in V, \\
0 \quad \text { if } x \notin V
\end{array}\right.
$$

is Lipschitz and $C^{p}$-smooth. Moreover, $K$ and $K_{0}$ are equal on the open set

$$
\left\{x \in X: \rho(x)<r_{1}\right\},
$$

which contains the subspace $Y$. Therefore $|K(x)-F(x)|=\left|K_{0}(x)-F(x)\right| \leq \varepsilon$ for every $x \in Y$. In particular, we have that

$$
|K(x)-f(x)|=|K(x)-F(x)| \leq \varepsilon,
$$

for each $x \in U \cap Y$.

To finish the proof of Theorem [1] it only remains to show Claim 5.

Proof of Claim 5. Let us take two Lipschitz functions $\xi_{1}, \xi_{2} \in C^{\infty}(\mathbb{R},[0,1])$ such that

$$
\begin{aligned}
& \xi_{1}^{\prime} \geq 0, \xi_{1}^{-1}(0)=(-\infty, \delta / 2], \xi_{1}^{-1}(1)=[\delta, \infty) \\
& \xi_{2}^{\prime} \leq 0, \xi_{2}^{-1}(1)=(-\infty, \delta / 4], \xi_{2}^{-1}(0)=[\delta / 2, \infty) .
\end{aligned}
$$

For every $j \in \mathbb{N}$ and every $x \in X$, we define

$$
f_{j}(x)=\xi_{1}\left(\mu_{E}\left(x-x_{j}\right)\right), \quad g_{j}(x)=\xi_{2}\left(\mu_{E}\left(x-x_{j}\right)\right) .
$$

Since $\xi_{1}, \xi_{2}$ and $\mu_{E}$ are Lipschitz, it follows that $\left\{f_{j}: j \in \mathbb{N}\right\} \cup\left\{g_{j}: j \in \mathbb{N}\right\}$ is a uniformly Lipschitz family. Moreover, all the functions $f_{j}$ and $g_{j}$ are $C^{p}$ smooth (notice that $\xi_{1}$ and $\xi_{2}$ are constant on a neighborhood of the origin). Let $\Phi_{1}: X \rightarrow c_{00} \subset c_{0}$ be the zero function. For every $j \geq 2$, let us define $\Phi_{j}: X \rightarrow c_{00}$ by

$$
\Phi_{j}(x)=\left(g_{1}(x), \ldots, g_{j-1}(x), 0,0, \ldots\right) .
$$

It is clear that $\left\{\Phi_{j}: j \in \mathbb{N}\right\}$ is uniformly Lipschitz, and each $\Phi_{j}$ is $C^{p}$-smooth. Take a $C^{\infty}$-smooth function $\eta: \mathbb{R} \rightarrow[0, \infty)$ such that $\eta$ is non-decreasing, $\eta(t)=t$ if $t \geq 1$, and $\eta=1 / 2$ on a neighborhood of the origin.

For each $j \in \mathbb{N}$, we define $S_{j}: X \rightarrow[0, \infty)$ by

$$
S_{j}(x)=f_{j}(x)+\eta\left(\left\|\Phi_{j}(x)\right\|\right) .
$$

Bearing in mind that $f_{j}$ and $\Phi_{j}$ are $C^{p}$-smooth, $\eta$ is $C^{\infty}$-smooth, $\|\cdot\|$ is a $C^{\infty}$-smooth norm, and $\eta$ is constant on a neighborhood of the origin, it follows that $S_{j}$ is a $C^{p}$ smooth function. Moreover, taking into account that $\left\{f_{j}: j \in \mathbb{N}\right\} \cup\left\{\Phi_{j}: j \in \mathbb{N}\right\}$ is a uniformly Lipschitz family, and that $\eta$ and $\xi$ are Lipschitz, it follows that $\left\{S_{j}: j \in \mathbb{N}\right\}$ is a uniformly Lipschitz family of non-negative $C^{p}$-smooth functions defined on $X$. 
Let $h \in C^{\infty}(\mathbb{R},[0,1])$ be a Lipschitz function such that $h$ is non-increasing and $h^{-1}(1)=(-\infty, 1 / 2]$ and $h^{-1}(0)=[1, \infty)$. We define

$$
\Psi_{j}=h \circ S_{j},
$$

for each $j \in \mathbb{N}$. It is patent that $\left\{\Psi_{j}: j \in \mathbb{N}\right\}=\left\{h \circ S_{j}: j \in \mathbb{N}\right\} \subset C^{p}(X,[0,1])$ is a uniformly Lipschitz family. Therefore, the collection $\left\{\Psi_{j}: j \in \mathbb{N}\right\}$ satisfies $(i)$ of Claim 5. Let us prove that $\left\{\Psi_{j}: j \in \mathbb{N}\right\}$ also satisfies $(i i),(i i i)$ and $(i v)$.

(ii) Pick $x \in V$. Choose $j_{x} \in \mathbb{N}$ and $r>0$ such that $W_{x}:=B\left(x, r ; \mu_{E}\right) \subset$ $B\left(x_{j_{x}}, \frac{\delta}{8} ; \mu_{E}\right)$. If $y \in W_{x}$, then $\mu_{E}\left(y-x_{j_{x}}\right)<\frac{\delta}{8}$ and $g_{j_{x}}(y)=1$. Therefore, for each $j>j_{x}$, we have that $\left\|\Phi_{j}(y)\right\| \geq\left\|\Phi_{j}(y)\right\|_{\infty} \geq\left|g_{j_{x}}(y)\right|=1$. Hence $\eta\left(\left\|\Phi_{j}(y)\right\|\right)=$ $\left\|\Phi_{j}(y)\right\| \geq 1$ and $S_{j}(y) \geq 1$. It follows from the definitions of the function $h$ that $\Psi_{j}(y)=h\left(S_{j}(y)\right)=0$ for each $y \in W_{x}$ and $j>j_{x}$.

(iii) Let us fix $x \in V$. Notice that $V \subset \bigcup_{j \in \mathbb{N}} B\left(x_{j}, \frac{\delta}{2} ; \mu_{E}\right)$. If $x \in B\left(x_{1}, \frac{\delta}{2} ; \mu_{E}\right)$, then $f_{1}(x)=0=\left\|\Phi_{1}(x)\right\|$ and $\eta\left(\left\|\Phi_{1}(x)\right\|\right)=1 / 2$. If $x \notin B\left(x_{1}, \frac{\delta}{2} ; \mu_{E}\right)$, there exists $j_{0}>1$ such that $x \in B\left(x_{j_{0}}, \frac{\delta}{2} ; \mu_{E}\right) \backslash \bigcup_{i<j_{0}} B\left(x_{i}, \frac{\delta}{2} ; \mu_{E}\right)$. From the definition of the functions $f_{j}, g_{j}$ and $\eta$, it follows that $f_{j_{0}}(x)=0$ and $\eta\left(\left(\left\|\Phi_{j_{0}}(x)\right\|\right)\right)=\frac{1}{2}$. Therefore, for each $x \in V$, there exists $j_{0} \in \mathbb{N}$ such that $\Psi_{j_{0}}(x)=h\left(S_{j_{0}}(x)\right)=h(1 / 2)=1$.

(iv) Take $x \in V$ and $j \in \mathbb{N}$. Assume that $\mu_{E}\left(x-x_{j}\right) \geq \delta$. From the definition of $f_{j}$, it follows that $f_{j}(x)=\xi_{1}\left(\mu_{E}\left(x-x_{j}\right)\right)=1$. Hence $S_{j}(x)=f_{j}(x)+\eta\left(\left\|\Phi_{j}(x)\right\|\right) \geq$ $f_{j}(x)=1$ and $\Psi_{j}(x)=h\left(S_{j}(x)\right)=0$.

An application. As an illustration of the usefulness of our main result, let us mention that D. Azagra and A. Montesinos have used Theorem 1 to show that, for every infinite-dimensional Banach space $X$ with a separable subspace $Y$ so that $X$ and $X / Y$ have $C^{p}$-smooth Lipschitz bumps, there exist $C^{p}$-diffeomorphisms deleting points with bounded supports, that is, there exist $C^{p}$-diffeomorphisms $h: X \rightarrow X \backslash\{0\}$ such that $h(x)=x$ if $\|x\| \geq 1$; see [1] for details.

Some related open questions. (1) Is it always true that if $X$ has a $C^{p}$-smooth Lipschitz bump and $Y \subset X$ is separable, then the quotient $X / Y$ has a $C^{p}$-smooth bump as well?

(2) Is " $X / Y$ has a $C^{p}$-smooth Lipschitz bump" necessary in Theorem 1]?

(3) Does Theorem 1 hold true when $f$ is not bounded?

(4) Is Theorem 1 true if we drop the Lipschitz condition everywhere?

(5) If $X$ has a separating polynomial, can we obtain real-analytic perturbed extensions $F$ ?

(6) What happens when $Y$ is not separable?

\section{REFERENCES}

[1] D. Azagra and A. Montesinos, Starlike bodies and diffeomorphisms deleting points in Banach spaces, to appear in Extracta Math.

[2] Y. Benyamini and J. Lindenstrauss, Geometrical Nonlinear Functional Analysis, Volume I, Amer. Math. Soc. Colloq. Publ., Vol. 48, Amer. Math. Soc., Providence, 2000. MR1727673 (2001b:46001)

[3] M. Cepedello, Approximation of Lipschitz functions by $\Delta$-convex functions in Banach spaces. Israel J. Math. 106 (1998), 269-284. MR.1656905 (99j:46016)

[4] R. Deville, V. Fonf, P. Hájek, Analytic and polyhedral approximation of convex bodies in separable polyhedral Banach spaces. Israel J. Math. 105 (1998), 139-154. MR1639743 (99h:46006)

[5] R. Deville, V. Fonf, P. Hájek, Analytic and $C^{k}$ approximations of norms in separable Banach spaces. Studia Math. 120 (1996), no. 1, 61-74. MR1398174|(97h:46012) 
[6] R. Deville, G. Godefroy, and V. Zizler, Smoothness and renormings in Banach spaces, vol. 64, Pitman Monographies and Surveys in Pure and Applied Mathematics, 1993. MR 1211634 (94d:46012)

[7] R. Fry, Approximation by functions with bounded derivative on Banach spaces, to appear in Bull. Australian Math. Soc. MR 2040055

[8] M. Leduc, Densité de certaines familles d'hyperplans tangents, C. R. Acad. Sci. (Paris) Ser. A 270 (1970), 326-328. MR0276733(43:2473)

Departamento de Análisis Matemático, Facultad de Ciencias Matemáticas, UniversiDad Complutense, 28040 Madrid, Spain

E-mail address: daniel_azagra@mat.ucm.es

Department of Mathematics and Computer Science, St. Francis Xavier University, P.O. Box 5000, Antigonish, Nova Scotia, Canada B2G 2W5

E-mail address: rfry@stfx.ca

Departamento de Análisis Matemático, Facultad de Ciencias Matemáticas, UniversiDad Complutense, 28040 Madrid, Spain

E-mail address: a_montesinos@mat.ucm.es 